\title{
Cost Analysis of Mobility Management Entities for SIGMA
}

\author{
Md. Shohrab Hossain \\ Mohammed Atiquzzaman \\ University of Oklahoma, Norman, OK 73019 \\ Email: \{shohrab, atiq\}@ou.edu
}

\author{
William Ivancic \\ NASA Glenn Research Center \\ Cleveland, OH 44135 \\ Email: wivancic@grc.nasa.gov
}

\begin{abstract}
To facilitate mobile computing, mobility protocols have been proposed and mobility management involves signaling costs. Widespread use of IP-enabled mobile devices have resulted in increase in number of mobile users in the network and the signaling cost on underlying mobility entities have increased significantly, which will result in performance degradation of the mobility protocols. However, there has been no comprehensive cost analysis of mobility protocol entities that considers all possible costs for mobility management. In this paper, we have developed analytical models to estimate total costs of mobility management entities of SIGMA, an IP-diversity based seamless handover protocol. We have presented numerical results to demonstrate the impact of increased of network size, mobility rate, traffic rate and data volume on these costs. Our results show that a significant amount of resources are required by the mobility entities for transmission, processing of various signaling messages, as well as searching location database. Our cost analysis will thus help network engineers estimate actual resource requirements of the network in future design.

Index Terms-Mobility Protocols, Analytical Modeling, Signaling cost, Seamless Handover
\end{abstract}

\section{INTRODUCTION}

Increasing demand for mobility in wireless data networks has given rise to various mobility management schemes. IETF proposed Mobile IPv6 (MIPv6) [1] support mobility of mobile hosts. But it has a number of drawbacks, such as, high handover latency, packet loss, inefficient routing path, etc. To address these drawbacks, seamless IP-diversity based mobility protocol, SIGMA [2] has been proposed.

In a mobile computing environment, a number of network parameters (such as, network size, mobility rate, traffic rate) influence signaling costs relating to mobility. These costs include overhead related to location update message, binding update message, DHCP request-reply messages and location query-reply messages etc. Widespread use of IP-enabled mobile devices have resulted in increase in number of mobile users in the network. This increased network size incurs more signaling costs on different mobility entities e.g., Location Manager (DNS), Access Router, Mobile Hosts, Correspondent Nodes etc. The huge amount of signaling was not expected earlier. Hence, mobility protocols must be analyzed with respect to their costs on various mobility management entities to ensure their smooth operation with increased load.

The research work reported in this paper was supported by NASA Grant NNX06AE44G.
There has been earlier attempts for signaling cost analysis ( [3], [4], [5], [6]) of mobility protocols. Fu et al. [4] analyze the signaling costs of SIGMA and HMIPv6. Xie et al. [3] perform cost analysis of Mobile IP to minimize the signaling cost while introducing a novel regional location management scheme. Makaya et al. [6] present an analytical model for the cost analysis of IPv6-based mobility protocols. Reaz et al. [5] perform the signaling cost analysis of SINEMO and NEMO BSP. However, these analysis did not consider all possible costs (e.g. query message, DHCP messages, SCTP address reconfiguration messages, etc.) and they did not compute the signaling costs on various mobility entities.

Most of the signaling cost analysis [4], [5] used random waypoint mobility model where mobile hosts pick a random speed and direction, resulting in sharp turns and sudden stops frequently, which is very unusual in real scenarios. Therefore, those work cannot accurately estimate the amount of resources required by the mobility entities. The main differences of this work are that we have considered all possible costs on various mobility management entities of SIGMA protocol, and have used city section mobility model, a realistic mobility model to compute the total costs on them.

The objective of this work is to analyze the signaling costs on various mobility entities of SIGMA mobility protocols and figure out how those costs are affected by network size, mobility rate, traffic rate, and data volume.

The contributions of this work are: (i) developing a mathematical model to estimate total costs of various mobility management entities of SIGMA: location manager, mobile host, correspondent nodes, access router, and complete network and (ii) analyzing the impact of network size, mobility rate, traffic rate, and data volume on these costs.

The analytical cost model developed in this paper covers all possible costs required for mobility management (including data delivery) and can help network engineers in estimating the actual resources requirement of the key entities of the network with increased load in future.

The rest of the paper is organized as follows. In Section II, a brief description of SIGMA protocol is given. Section III explains city section mobility model. In Section IV, signaling costs of various mobility entities are derived for SIGMA. Section V analyzes the results. Finally, Section VI has the concluding remarks. 


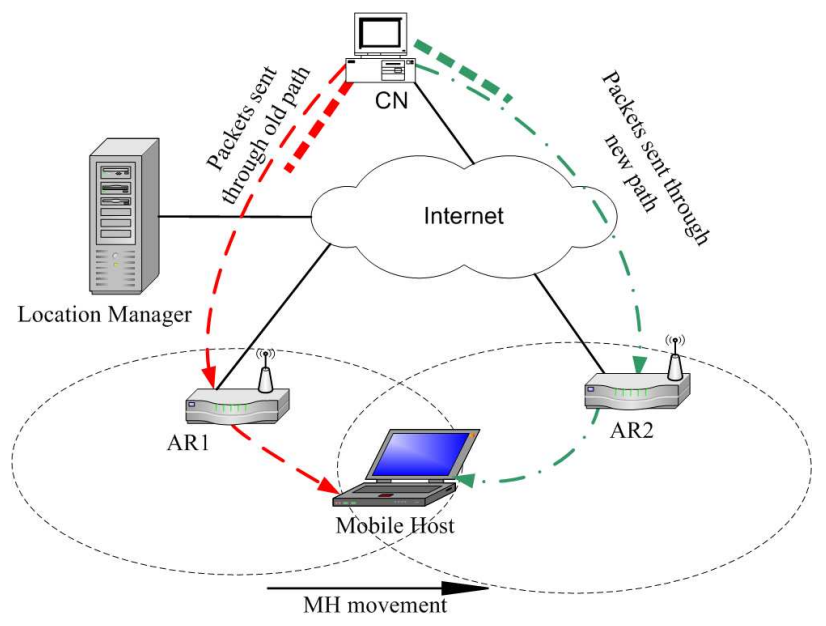

Fig. 1. SIGMA Architecture [2]

\section{SIGMA Mobility Protocol}

The architecture of SIGMA [2] is shown in Fig. 1. The Location Manager (LM) is responsible for keeping location database of mobile hosts. Whenever any Correspondent Node $(\mathrm{CN})$ wants to send data to a $\mathrm{MH}$, it must first send a query message to the LM to obtain its current IP address. Hence, every MH must send its new IP address in a network it has moved to the LM; these are termed as Location Updates. Moreover, every subnet crossing triggers binding updates. Each $\mathrm{MH}$ needs to send a binding update after handover to every $\mathrm{CN}$ it is communicating with. SIGMA achieves seamless handover by using the multi-homing feature of Stream Control Transport Protocol (STCP) and uses a make-before-break strategy (by obtaining a secondary IP address from the new Access Router) so that the ongoing communication is not interrupted during handover.

\section{City SECTION MOBILITY MODEL}

In this section, we explain the City Section Mobility (CSM) model that is used to simulate the movement pattern of the mobile hosts. The stochastic properties of CSM model has been analyzed in [7] and we present some results to obtain the residence time of $\mathrm{MH}$ roaming in the environment.

In CSM model, each mobile node starts at a predefined intersection of two streets. It then randomly chooses a destination. Moving to this destination involves (at most) one horizontal and one vertical movement. Upon reaching the destination, it pauses for some random time and the same process is repeated which is termed as epoch. Let the environment be a rectangular area of dimension $a \times b$ as shown in Fig. 2. The circles represent the radio coverage area of the Access Routers. There are $y$ rows of ARs and $x$ ARs in each row. Let there be $N_{s}$ horizontal roads (streets) and $N_{a}$ vertical roads (avenues) forming a particular section of a city. Therefore, the expected epoch length of CSM model can be obtained as follows [7]:

$$
E(L)=\frac{a\left(N_{a}+1\right)}{3 N_{a}}+\frac{b\left(N_{s}+1\right)}{3 N_{s}}
$$

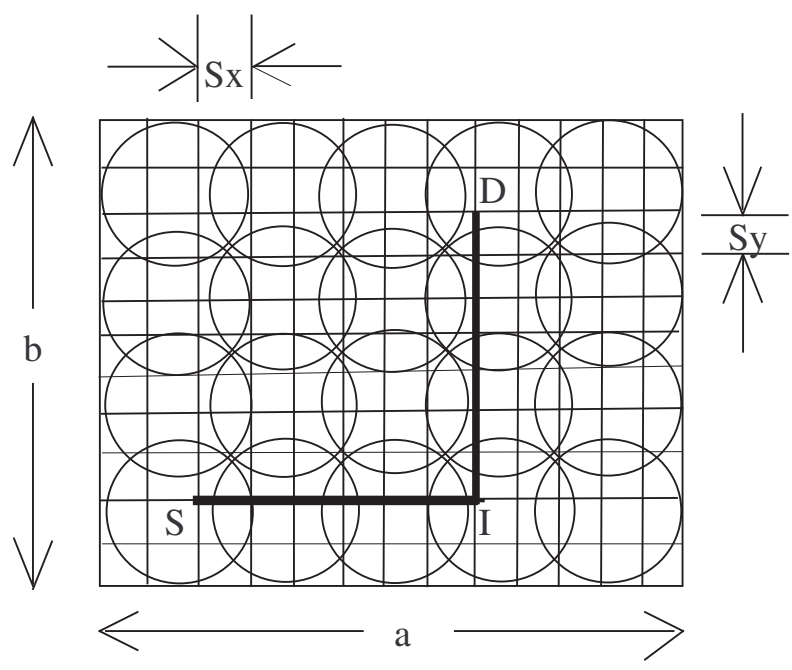

Fig. 2. Road network in CSM model.

Let the speed of the mobile host vary between $V_{x}^{\min }$ to $V_{x}^{\max }$ for some horizontal road segment and between $V_{y}^{\text {min }}$ to $V_{y}^{\max }$ for some vertical road segment. Therefore, the expected epoch time [7] is given by,

$E(T)=E\left(L_{x}\right) \frac{\ln \left(V_{x}^{\max } / V_{x}^{\min }\right)}{V_{x}^{\text {max }}-V_{x}^{\min }}+E\left(L_{y}\right) \frac{\ln \left(V_{y}^{\max } / V_{y}^{\min }\right)}{V_{y}^{\max }-V_{y}^{\min }}$

The expected number of subnet crossings in an epoch can be obtained as follows [7]:

$$
\begin{aligned}
E(C) & =\frac{x(x+1) K}{6 N_{a}^{2}}\left(6 N_{a}-4 x K+K+3\right) \\
& +\frac{y(y+1) K}{6 N_{s}^{2}}\left(6 N_{s}-4 y K+K+3\right)
\end{aligned}
$$

If the average pause time is $E(U)$, average residence time in a subnet can be estimated as follows [7]:

$$
T_{r}=\frac{E(T)+2 E(U)}{E(C)}
$$

The expression of subnet residence time $\left(T_{r}\right)$ will be used to compute the total costs in Section V.

\section{Cost Analysis}

In this section, we analyze the signaling cost of various mobility entities of SIGMA protocol: Location Manager, Mobile Host, Correspondent Node, Access Router, and Complete Network.

\section{A. Assumptions}

Following are assumptions for cost analysis:

- Session arrival rate for each mobile host is equal.

- The data (file) size in each session is equal.

- Uniform distribution of mobile hosts over the region of the network.

- Binary search is used to search location database. 


\section{B. Notations}

The notations to be used in the analysis are listed below.

$N_{m} \quad$ Number of Mobile Hosts,

$N_{c} \quad$ Average number of CNs with which a MH is communicating,

$\beta_{l} \quad$ Per hop transmission cost for location update,

$\beta_{q} \quad$ Per hop transmission cost for query message,

$\beta_{d} \quad$ Per hop transmission cost for DHCP request /reply message,

$\beta_{s} \quad$ Per hop transmission cost for SCTP address reconfiguration message,

$\beta_{d p} \quad$ Per hop transmission cost for average data packet,

$\beta_{d a}$ Per hop transmission cost for acknowledgement packet (for data packet),

$\sigma \quad$ Proportionality constant of signaling cost over wired and wireless link,

$\eta \quad$ Linear coefficient for lookup cost,

$T_{r} \quad$ Subnet residence time,

$\lambda_{s} \quad$ Average session arrival rate for each mobile host,

$x \quad$ Number of access routers in a row,

$y \quad$ Number of access routers in a column,

$\kappa \quad$ Maximum transmission unit,

$\alpha \quad$ Data (or file) size on each session,

$\chi \quad$ Average number of retransmission for each data and ACK packet.

$\Phi_{m l}$ Average number of hops between MH and LM,

$\Phi_{m c}$ Average number of hops between $\mathrm{MH}$ and $\mathrm{CN}$,

$\Phi_{l c} \quad$ Average number of hops between LM and CN,

$\delta_{l} \quad$ Processing cost at LM for each location update,

$\delta_{c} \quad$ Processing cost at $\mathrm{CN}$ for binding update message from $\mathrm{MH}$,

$\delta_{r} \quad$ Processing cost at $\mathrm{MH}$ to update local routing table,

$\delta_{a} \quad$ Processing cost at AR for DHCP request message.

\section{Location Manager}

The signaling costs on the Location Manager are due to location update messages sent by the MHs, and the query message about current location of the MH exchanged with CNs.

1) Location Updates: In SIGMA, every subnet crossing (that happens every $T_{r}$ seconds) by an $\mathrm{MH}$, triggers a location update message to be sent to LM. The LM receives each LU message, processes (updates) the corresponding entry, and sends the acknowledgement back to the MH. Thus, each location update requires transmission cost $\left(2 \beta_{l}\right)$ and processing cost $\left(\delta_{l}\right)$ at LM. We have considered $N_{l}$ number of LMs for the all the $N_{m}$ MHs in the network topology. So on the average, $N_{m}^{l}=N_{m} / N_{l}$ mobile hosts will send location updates to each LM. Hence, the signaling load on LM due to location updates is as follows:

$$
\Gamma_{L M}^{L}=N_{m}^{l} \frac{2 \beta_{l}+\delta_{l}}{T_{r}}
$$

2) Query Message: For each association between $\mathrm{MH}$ and $\mathrm{CN}$, query message about the current location of $\mathrm{MH}$ is sent to the LM, and the LM sends the reply to that query after looking up the table. This involves transmission cost and lookup cost. Since the session arrival rate is $\lambda_{s}$, for each association lookup cost is $\eta \lambda_{s} \log _{2}\left(N_{m} / N_{l}\right)$. Now for all the sessions between the MHs and CNs, the signaling load on LM is as follows:

$$
\Gamma_{L M}^{Q}=N_{m}^{l} N_{c}\left(2 \beta_{q} \lambda_{s}+\eta \lambda_{s} \log _{2}\left(N_{m} / N_{l}\right)\right)
$$

3) Total Cost on LM: The total cost on the LM can be obtained by adding Eqns. (5) and (6).

$$
\Gamma_{L M}=\Gamma_{L M}^{L}+\Gamma_{L M}^{Q}
$$

\section{Mobile Host}

The signaling costs on $\mathrm{MH}$ are due to the query messages exchanged with ARs, binding update messages exchanged with CNs, and location update messages sent to LM, and packet delivery.

1) DHCP request-reply Messages: When the $\mathrm{MH}$ approaches the radio coverage area of a new AR, it acquires new IP address from that AR by exchanging messages that involves transmission cost through the wireless media.

$$
\Gamma_{M H}^{D}=\frac{2 \sigma \beta_{d}}{T_{r}}
$$

2) Binding Update Message: After acquiring IP address, the $\mathrm{MH}$ notifies $\mathrm{CN}$ about the availability of new IP address through SCTP address dynamic reconfiguration option [8] (cost is $2 \sigma \beta_{s}$ ). When $\mathrm{MH}$ moves further into the radio coverage area of the new AR, it sends ASCONF message [8] to $\mathrm{CN}$, using which $\mathrm{CN}$ sets its primary destination (for $\mathrm{MH}$ ) to IP2 (cost of $2 \sigma \beta_{s}$ ). When the MH moves out from the coverage area of the previous AR, it sends another message to $\mathrm{CN}$ to delete previous IP address. The MH also updates its local routing table to make sure future outgoing packets are sent through new AR (requires processing cost of $\delta_{r}$ ).

$$
\Gamma_{M H}^{B}=N_{c} \frac{2 \sigma \beta_{s}+2 \sigma \beta_{s}+2 \sigma \beta_{s}+\delta_{r}}{T_{r}}
$$

3) Location Update Message: Every $\mathrm{MH}$ sends location update message to LM after every subnet crossing.

$$
\Gamma_{M H}^{L}=\frac{2 \sigma \beta_{l}}{T_{r}}
$$

4) Packet Delivery Cost: Each MH communicates with $N_{c}$ correspondent nodes. Since average session size is $\alpha$, number of packets in a session is $\left[\frac{\alpha}{\kappa}\right]$. There are $(\chi+1)$ attempts of transmission for each data (and ACK) packets. Hence, the packet delivery costs for each $\mathrm{MH}$ per second is

$$
\Gamma_{M H}^{P}=N_{c}\left\lceil\frac{\alpha}{\kappa}\right\rceil \sigma \lambda_{s}(\chi+1)\left(\beta_{d p}+\beta_{d a}\right)
$$

5) Total cost on $M H$ : Thus, the total cost on each MH can be obtained by adding Eqns. (7), (8), (9) and (10) as

$$
\Gamma_{M H}=\Gamma_{M H}^{D}+\Gamma_{M H}^{B}+\Gamma_{M H}^{L}+\Gamma_{M H}^{P}
$$




\section{E. Correspondent Node}

The cost on each $\mathrm{CN}$ are due to the query message exchanged with LM, binding update message exchanged with MHs, and packet delivery cost.

1) Query Message: Each $\mathrm{CN}$ sends query messages to the LM for each association with the MH per session time $\left(T_{s}\right)$.

$$
\Gamma_{C N}^{Q}=\frac{2 \beta_{q}}{T_{s}}
$$

2) Binding Update Message: $\mathrm{MH}$ notifies the $\mathrm{CN}$ after moving into the coverage area of a new AR which involves three SCTP ASCONF messages (add IP, set primary IP, and delete IP). Therefore transmission costs, and processing cost on $\mathrm{CN}$ are

$$
\Gamma_{C N}^{B}=\frac{6 \beta_{s}+\delta_{c}}{T_{r}}
$$

3) Packet Delivery Cost: In each session, a file of size $\alpha$ is transferred from the $\mathrm{CN}$ to the $\mathrm{MH}$. The packet delivery cost on $\mathrm{CN}$ per unit time can be obtained as

$$
\Gamma_{C N}^{P}=\lambda_{s}\left\lceil\frac{\alpha}{\kappa}\right\rceil(\chi+1)\left(\beta_{d p}+\beta_{d a}\right)
$$

4) Total Cost on each $C N$ : Therefore, the total cost on each $\mathrm{CN}$ can be obtained by adding Eqns. (11), (12) and (13):

$$
\Gamma_{C N}=\Gamma_{C N}^{Q}+\Gamma_{C N}^{B}+\Gamma_{C N}^{P}
$$

\section{F. Access Router}

Total cost on each AR is due to exchange of DHCP messages, binding updates, location updates, and packet delivery.

1) DHCP Messages: After every subnet crossing, DHCP request message is sent to the AR. If there are $x y$ ARs in the foreign network, average number of MHs under each AR is $N_{m} / x y$. Therefore, cost at AR due to DHCP request-reply messages are

$$
\Gamma_{A R}^{D}=\frac{N_{m}}{x y} \times \frac{2 \sigma \beta_{d}+\delta_{a}}{T_{r}}
$$

2) Binding Update Message: After acquiring new IP address, each $\mathrm{MH}$ notifies the $\mathrm{CN}$ (using three pairs of SCTP ASCONF messages) through the AR. Since average number of MHs under each AR is $N_{m} / x y$, and each MH communicates with $N_{c}$ CNs, therefore, cost associated with the binding update message for the AR is

$$
\Gamma_{A R}^{B}=\frac{N_{c} N_{m}}{x y} \times \frac{6 \sigma \beta_{s}}{T_{r}}
$$

3) Location Update Message: For each subnet crossing by the $\mathrm{MH}$, location update message is sent to the LM through the AR. So the costs at AR are

$$
\Gamma_{A R}^{L}=\frac{N_{m}}{x y} \times \frac{2 \sigma \beta_{l}}{T_{r}}
$$

4) Packet Delivery Cost: Each MH under an AR communicates with $N_{c}$ CNs, so the packet delivery cost on each AR

$$
\Gamma_{A R}^{P}=\frac{N_{m} N_{c}}{x y}\left\lceil\frac{\alpha}{\kappa}\right\rceil \sigma \lambda_{s}(\chi+1)\left(\beta_{d p}+\beta_{d a}\right)
$$

5) Total cost on each AR: Therefore, the total cost on each AR can be obtained by adding Eqns. (15), (16) and (17), and (18)

$$
\Gamma_{A R}=\Gamma_{A R}^{D}+\Gamma_{A R}^{B}+\Gamma_{A R}^{L}+\Gamma_{A R}^{P}
$$

\section{G. Complete Network}

In order to compute the total cost on the network as a whole, we will consider resources (such as, bandwidth, processing power etc) consumed due to the SIGMA mobility protocol.

1) Query Message: For each $\mathrm{MH}-\mathrm{CN}$ association, query messages are exchanged between $\mathrm{CN}$ and LM. Each $\mathrm{MH}$ has an average of $N_{c} \mathrm{CNs}$; therefore, total number of CNs for all the mobile hosts are $N_{m} N_{c}$. So the transmission cost of $N_{c} N_{m}\left(2 \Phi_{l c} \beta_{q}\right) \lambda_{s}$ and searching costs of $N_{c} N_{m}\left(\eta \lambda_{s} \log _{2} N_{m}\right)$ are required. Hence, the costs for the query messages,

$$
\Gamma_{N e t}^{Q}=N_{m} N_{c} \lambda_{s}\left(2 \beta_{q} \Phi_{l c}+\eta \log _{2} N_{m}\right)
$$

2) Location Update Message: Each subnet crossing of $\mathrm{MH}$ triggers location update to be sent to LM which processes the message and sends acknowledgement back to MH. Therefore, costs of location updates on the network are

$$
\Gamma_{\text {Net }}^{L}=N_{m} \frac{2\left(\Phi_{m l}-1+\sigma\right) \beta_{l}+\delta_{l}}{T_{r}}
$$

3) DHCP request-reply message: The costs associated with the DHCP request-reply messages are

$$
\Gamma_{N e t}^{D}=N_{m} \frac{2 \sigma \beta_{d}+\delta_{a}}{T_{r}}
$$

4) Binding Updates: After each subnet crossing of each $\mathrm{MH}$, binding updates are sent to all the correspondent nodes. The binding update is proportional to the distance (in hops) between the $\mathrm{MH}$ and $\mathrm{CN}$.

$$
\Gamma_{N e t}^{B}=N_{m} N_{c} \frac{6\left(\Phi_{m c}-1+\sigma\right) \beta_{s}+\delta_{c}}{T_{r}}
$$

5) Packet Delivery Cost: After getting the IP address of the $\mathrm{MH}$ from LM, the $\mathrm{CN}$ send data packets directly to the $\mathrm{MH}$ through $\Phi_{m c}-1$ wired hops and one wireless hop. Hence packet delivery cost for all the communications in foreign network can be obtained as:

$\Gamma_{N e t}^{P}=N_{m} N_{c} \lambda_{s}\left\lceil\frac{\alpha}{\kappa}\right\rceil(\chi+1)\left(\beta_{d p}+\beta_{d a}\right)\left(\Phi_{m c}-1+\sigma\right)$

6) Total Cost on the Network: Thus, the total cost on the complete network due to SIGMA protocol can be obtained by adding Eqns. (20), (21), (22), (23) and (24):

$$
\Gamma_{N e t}=\Gamma_{N e t}^{Q}+\Gamma_{N e t}^{L}+\Gamma_{N e t}^{D}+\Gamma_{N e t}^{B}+\Gamma_{N e t}^{P}
$$




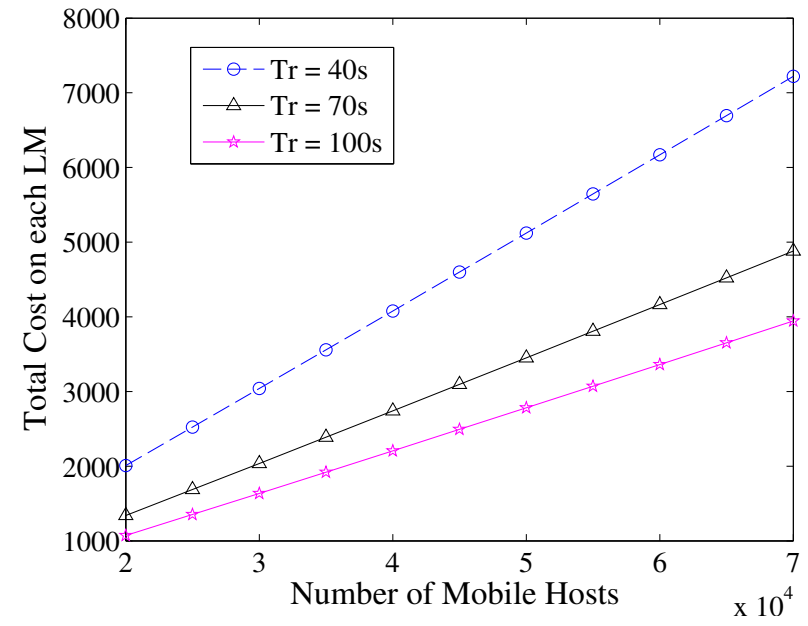

Fig. 3. Total cost on each LM vs. number of mobile hosts for different residence time

\section{Numerical Results}

In this section, the expressions derived in the cost analysis section are used to find out total cost on various entities of SIGMA protocols: $\mathrm{LM}, \mathrm{MH}, \mathrm{CN}$, AR, and complete network. The parameters that affect the total cost are number and speed of MHs, number of CNs, session arrival rate, session size, session to mobility ratio (defined as $T_{r} \times \lambda_{s}$ ).

Parameters relating to the CSM model are obtained from the previous works [7]: $a=36 \mathrm{~km}, b=24 \mathrm{~km}, r=0.5 \mathrm{~km}, x$ $=51, y=34, K=5, V_{x}^{\max }=V_{y}^{\max }=120 \mathrm{~km} / \mathrm{hr}, V_{x}^{\min }=$ $V_{y}^{\min }=25 \mathrm{~km} / \mathrm{hr}$, and $E(U)=4 \mathrm{sec}$. Using these parameter values, subnet residence times of MHs are computed using Eqn. (4) in Sec. III. Parameters relating to mobility protocols are similar to previous works [3], [4], [5]: $N_{c}=5 ; N_{l}=10$, $N_{m}=40000, \beta_{l}=0.6, \beta_{s}=0.6, \beta_{q}=0.6, \beta_{d}=1.4, \beta_{d p}=$ 5.72, $\beta_{d a}=0.60, \sigma=10, \eta=0.3, T_{r}=70, \lambda_{s}=0.01, \Phi_{m l}$ $=35, \Phi_{m c}=35, \Phi_{l c}=35, \delta_{l}=30, \delta_{r}=5, \delta_{a}=30, \delta_{c}=30$, $x=51, y=34, \kappa=512, \alpha=10240, \chi=3$.

\section{A. $L M$}

In Fig. 3, the impact of number of MHs on the total cost of each LM are shown for $T_{r}=40,70,100 \mathrm{~s}$. It is found that total cost increases for each LM for higher number of MHs and lower residence time as more updates are sent to the LM.

Fig. 4 shows the effect of SMR on the total cost of each LM for different number of MHs. Results show that total cost for each LM reduces with higher SMR.

\section{B. $M H$}

Fig. 5 shows the total cost on each $\mathrm{MH}$ as a function of number of CNs for various $\lambda_{s}$. As number of CNs and $\lambda_{s}$ increase, more binding updates and data are sent with higher rates. Therefore, total cost on each MH goes up.

\section{C. $C N$}

In Fig. 6, it is found that total cost on each $\mathrm{CN}$ remains constant with increased $\mathrm{MH}$ speed due to the dominance of

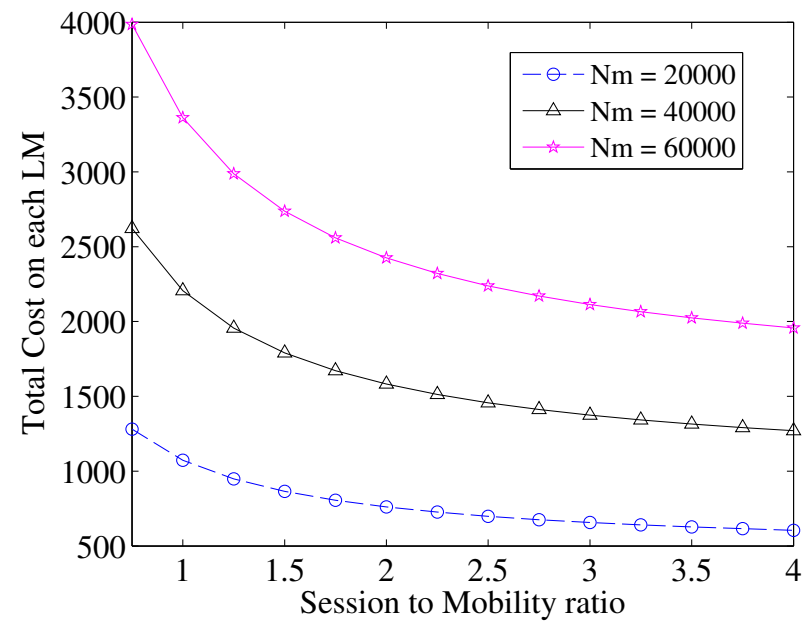

Fig. 4. Total cost on each LM vs. session to mobility ratio for different number of mobile hosts

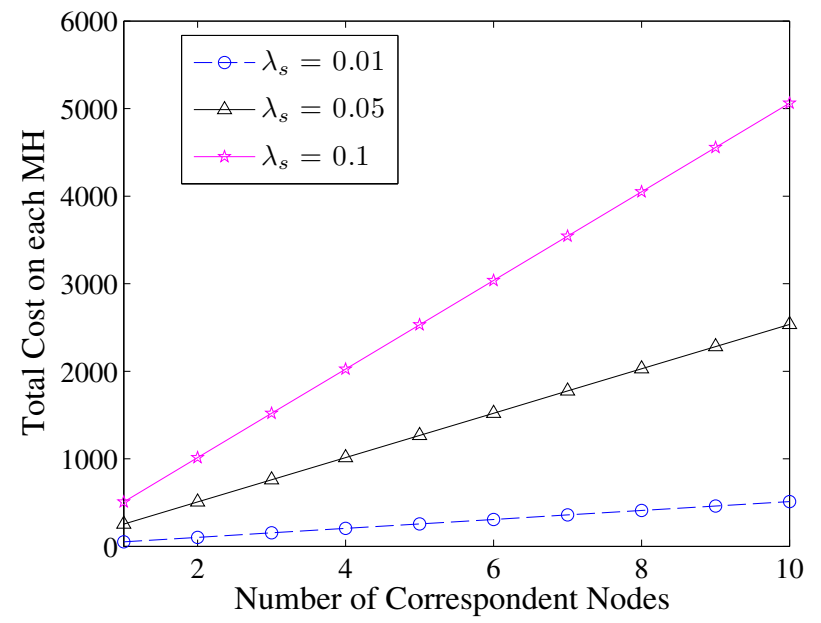

Fig. 5. Total cost on each $\mathrm{MH}$ vs. number of correspondent nodes for different values of $\lambda_{s}$ and $\chi$

the packet delivery term. However, when network is congested, packets get corrupted and number of retransmissions goes up which produces more cost on each $\mathrm{CN}$.

D. $A R$

Fig. 7 shows that total cost for each $\mathrm{AR}$ increases for increased number of MHs and session arrival rate as AR has to process and transmit more data and signaling packets.

\section{E. Complete Network}

In Fig. 8, total cost for the network is shown as a function of number of MHs for different values of $\Phi_{m c}, \Phi_{l c}$, and $\Phi_{m l}$. It shows that total cost is invariant of $\Phi_{l c}$ and $\Phi_{m l}$ but depends on $\Phi_{m c}$, i.e., average number of hops between $\mathrm{MH}$ and $\mathrm{CN}$, which is the length of the path of the data packets.

Fig. 9 shows that higher value of $N_{c}$ produces more cost in the network; it further rises for higher $\lambda_{s}$ and $\chi$, as more sessions produce more packets making network congested. 


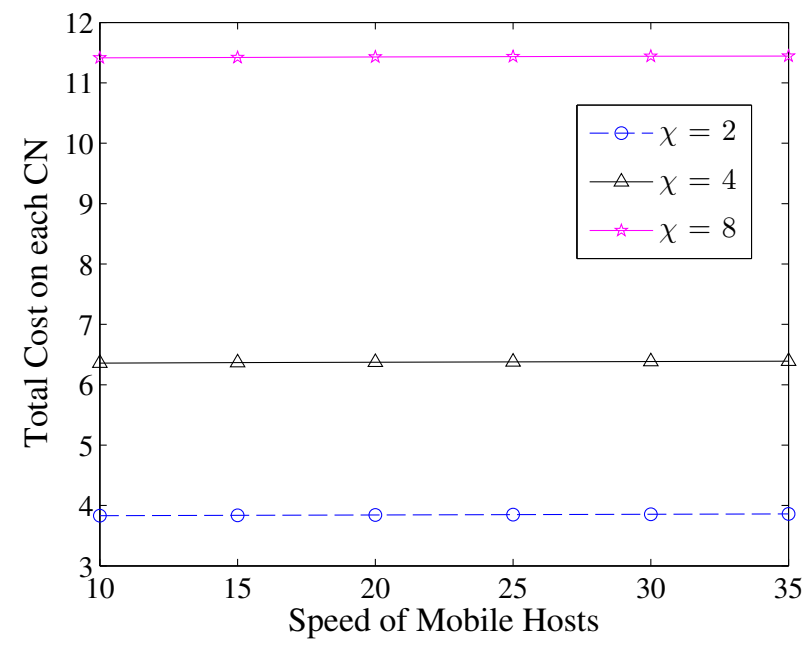

Fig. 6. Total cost on each $\mathrm{CN}$ vs. speed of mobile hosts for different values of $\chi$

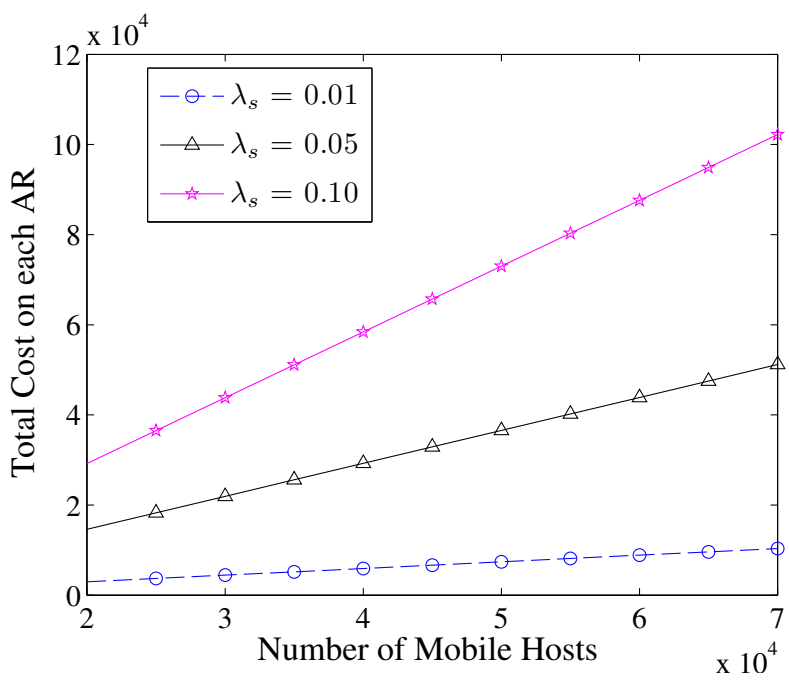

Fig. 7. Total cost on each AR vs. number of mobile hosts for different session arrival rate

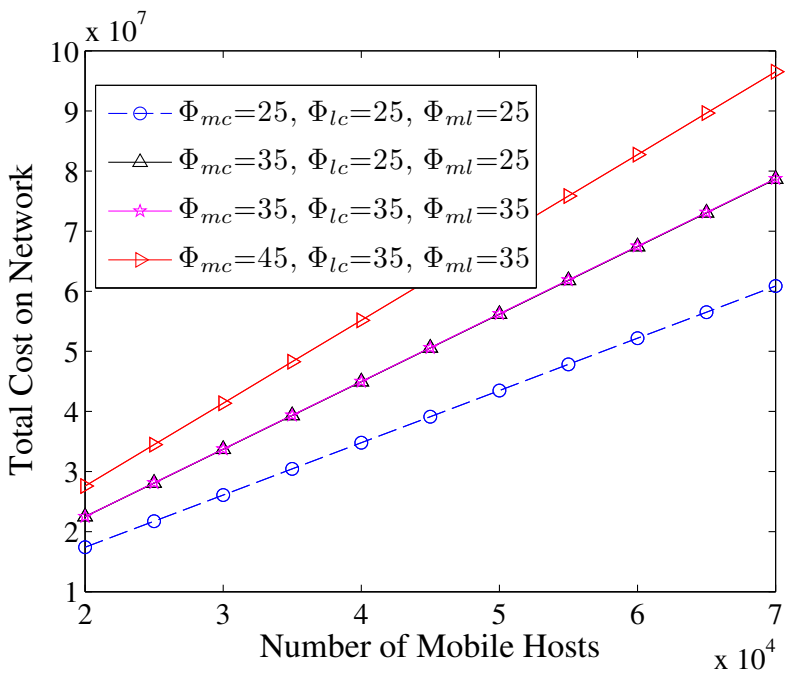

Fig. 8. Total cost on the network vs. number of mobile hosts for different values of $\Phi_{m c}, \Phi_{l c}$ and $\Phi_{m l}$

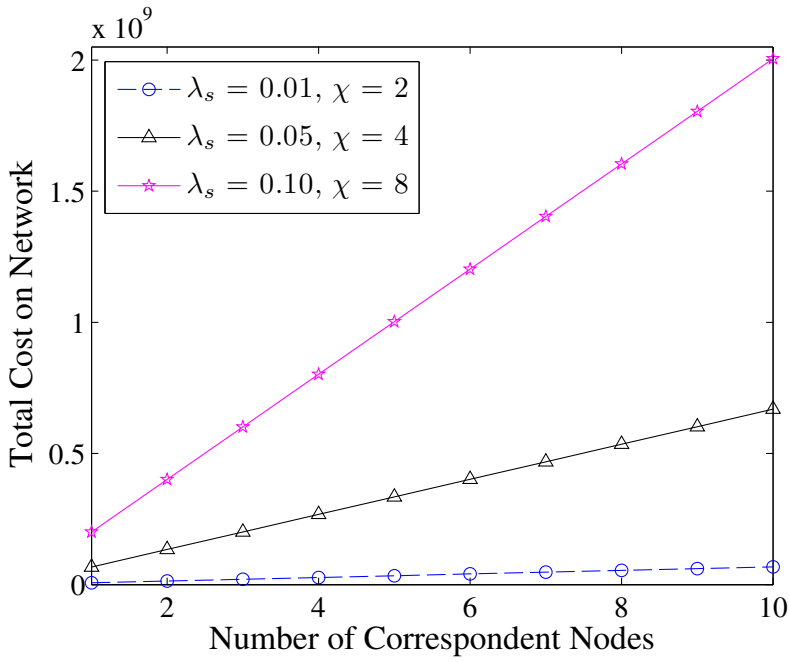

Fig. 9. Total cost on the network vs. number of $\mathrm{CNs}$ for different values of $\lambda_{s}$ and $\chi$

\section{CONCLUSION}

In this paper, we have developed analytical models to compute total costs on various mobility management entities of SIGMA, an IP-diversity based mobility protocol. We have presented numerical results and analyzed the impact of increased network size, mobility rate and traffic rate on these entities. Results show that data packet delivery costs dominate other signaling costs. However, a significant amount of resources are required by the mobility entities to transmit, process various signaling messages, search location database etc. Since the network was not originally designed to handle such expansion in mind, our comprehensive cost analysis will help network engineers in estimating the actual resource requirements by key entities of the network in future design.

\section{REFERENCES}

[1] D. Johnson, C. E. Perkins, and J. Arkko, "Mobility support in IPv6," IETF RFC 3775, June 2004

[2] S. Fu and M. Atiquzzaman, "SIGMA: A Transport Layer Handover Protocol for Mobile Terrestrial and Space Networks," e-Business and Telecommunication Networks, Springer, pp. 41-52, 2006.

[3] J. Xie and I. Akyildiz, "A novel distributed dynamic location management scheme for minimizing signaling costs in Mobile IP," IEEE Transactions on Mobile Computing, vol. 1, no. 3, pp. 163-175, July 2002.

[4] S. Fu and M. Atiquzzaman, "Signaling cost and performance of SIGMA: A seamless handover scheme for data networks," Wireless Communication and Mobile Computing, vol. 5, no. 7, pp. 825-845, Nov 2005.

[5] A. S. Reaz, P. K. Chowdhury, and M. Atiquzzaman, "Signaling cost analysis of SINEMO: Seamless End-to-End Network Mobility," in First ACM/IEEE International Workshop on Mobility in the Evolving Internet Architecture, San Francisco, CA, Dec 01, 2006, pp. 37-42.

[6] C. Makaya and S. Pierre, "An analytical framework for performance evaluation of ipv6-based mobility management protocols," IEEE Transactions on Wireless Communications, vol. 7, no. 3, pp. 972-983, March 2008.

[7] M. S. Hossain and M. Atiquzzaman, "Stochastic properties and application of city section mobility model," in IEEE Global Communications Conference (GLOBECOM), Honolulu, HI, Nov 30- Dec 42009.

[8] R. Stewart, M. Ramalho, and Q. X. et al., "Stream control transmission protocol (stcp) dynamic address reconfiguration," IETF DRAFT draftietf-tsvwq-addip-sctp-09.txt, June 2004. 